\title{
Noncollinear coupling of iron layers through native iron oxide spacers
}

\author{
Thomas Diederich, Sebastien Couet, and Ralf Röhlsberger \\ Hamburger Synchrotronstrahlungslabor (HASYLAB), Deutsches Elektronen-Synchrotron (DESY), Notkestraße 85, 22603 Hamburg, \\ Germany
}

(Received 21 May 2007; revised manuscript received 4 July 2007; published 1 August 2007)

\begin{abstract}
We have found a magnetic superstructure in multilayers that consist of iron and its native oxide. Employing nuclear resonant scattering of synchrotron radiation from ${ }^{57} \mathrm{Fe}$ probe layers, this manifests as $1 / 2$-order Bragg peaks in the reflectivity curve. From the field dependence of their intensity, we deduce the existence of two magnetic sublattices that are canted by an angle of almost $90^{\circ}$ and rotate as one unit in low fields. For higher fields, the two sets of moments gradually align collinear to the external field. This behavior can be explained by a coupling of two adjacent Fe layers that is mediated by an antiferromagnetic order in the oxide spacer between them.
\end{abstract}

DOI: 10.1103/PhysRevB.76.054401

PACS number(s): 75.70.Cn, 61.10.Kw, 75.25.+z, 76.80.+y

\section{INTRODUCTION}

The discovery of oscillatory interlayer coupling of ferromagnetic layers through nonmagnetic spacers has initiated a multitude of activities in fundamental and applied research. Meanwhile, also the properties of multilayered systems consisting of directly coupled magnetic layers have found increasing interest due to their rich landscape of magnetic phases. ${ }^{1}$ Relatively unexplored systems are heterostructures that are formed of a magnetic material and its native oxide. These layer systems were recently proposed as a new material with high magnetization and low electrical conductivity. ${ }^{2}$ The magnetic order, however, that develops in such systems and leads to their remarkable magnetic properties remained rather unexplored.

In this paper, we report on the magnetic structure of polycrystalline multilayers consisting of $\mathrm{Fe}$ and its native oxide. Remarkably, we find a magnetic superstructure with an almost orthogonal moment alignment between adjacent Fe layers that are separated by an ultrathin oxide layer. The magnetic structure could be revealed by employing nuclear resonant scattering (NRS) of synchrotron radiation from ultrathin ${ }^{57} \mathrm{Fe}$ probe layers embedded in the layer system. ${ }^{3-5}$ Samples are typically illuminated under grazing angles of a few milliradians and the specularly reflected signal is recorded. The magnetic hyperfine interaction renders this technique very sensitive to the magnitude and the direction of magnetic fields at the position of the ${ }^{57} \mathrm{Fe}$ nuclei. These parameters become accessible via the analysis of temporal beat patterns in the reflected signal that is recorded after pulsed excitation by synchrotron radiation. Analyzing this signal as a function of momentum transfer, the method allows one to determine the depth profile of the magnetic moment orientation. For instance, magnetic superstructures in periodic layer systems manifest as additional Bragg peaks that appear between the structural Bragg peaks, ${ }^{6}$ similar as in polarized neutron reflectometry.

\section{EXPERIMENTS}

In this experiment, Fe layers with a thin $(0.6 \mathrm{~nm}){ }^{57} \mathrm{Fe}$ probe layer embedded were prepared by dc sputter deposi- tion in a UHV chamber on $\mathrm{Si}(100)$ substrates. Native Feoxide layers were formed at room temperature via exposure to $6500 \mathrm{~L}\left(1 \mathrm{~L}=10^{-6}\right.$ Torr s) oxygen at a pressure of about $5 \times 10^{-5}$ mbar. Under these conditions, a $1.6 \mathrm{~nm}$ thick film of $\mathrm{Fe}$ oxide forms. Multilayers were then obtained by alternating deposition of $\mathrm{Fe}$ and subsequent exposure to oxygen. A terminating Al layer $(4 \mathrm{~nm})$ was used for protection against oxidation in ex situ experiments.

The experiments have been carried out at the Nuclear Resonance beamline ID18 of the European Synchrotron Radiation Facility (ESRF). ${ }^{7}$ A series of time spectra was recorded in situ to follow the evolution from a single iron layer over an $\mathrm{Fe} / \mathrm{Fe}$-oxide bilayer to a trilayer $(\mathrm{Fe}[1.7 \mathrm{~nm}] / \mathrm{Fe}$ oxide$[1.6 \mathrm{~nm}] / \mathrm{Fe}[2.5 \mathrm{~nm}])$, as shown in Figs. 1(a)-1(c), respectively. In these measurements, an external field of $\vec{H}=700$ Oe was oriented parallel to the photon wave vector $\vec{k}_{0}$. The incident angle was kept at $\varphi=0.24^{\circ}$, which is above the critical angle of $\mathrm{Fe}\left(\varphi_{c}=0.22^{\circ}\right)$ and ensures that the whole depth of the sample is probed.

\section{RESULTS}

The beat pattern in Fig. 1(a) is characteristic for a parallel alignment of the Fe layer magnetization with $\vec{k}_{0}$, and its period corresponds to a magnetic hyperfine field of $32.6 \mathrm{~T}$ at the nuclei. This situation does not change if the Fe layer is oxidized, as shown in Fig. 1(b). Even though the oxide is in very close proximity $\left(0.6 \mathrm{~nm}\right.$ apart) to the probing ${ }^{57} \mathrm{Fe}$ layer, neither the magnitude of the hyperfine field nor its orientation is affected. This proves the very sharp boundary between the Fe metal and its native oxide. However, covering this bilayer with another iron layer (with ${ }^{57} \mathrm{Fe}$ embedded) leads to a significant change in the time spectrum as shown in Fig. 1(c). The envelope of the beats is no longer exponential, but the intensity ratios of neighboring peaks exhibit an extra modulation. This can be attributed to a noncollinear alignment of the magnetic hyperfine fields in the two probe layers with the external magnetic field. From the data, one derives a coupling angle between the magnetic moments of these layers of $60^{\circ} \pm 5^{\circ}$, which is symmetric about the direction of the external field. 


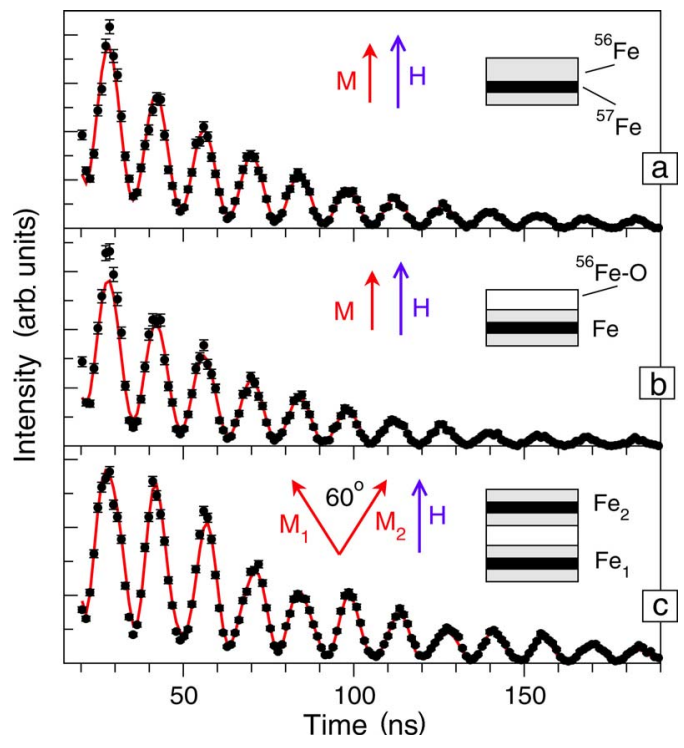

FIG. 1. (Color online) Time spectra of NRS at three different stages of the growth of an $\mathrm{Fe} / \mathrm{Fe}$-oxide/Fe trilayer. The solid lines are theoretical simulations from which the magnetization direction in the ${ }^{57} \mathrm{Fe}$ probe layers relative to the external magnetic field of $\vec{H}=70 \mathrm{mT}$ was deduced. While for a single iron layer the magnetization is parallel to $\vec{H}$, the two magnetic moments in the trilayer are canted by an angle of about $60^{\circ}$.

Of particular interest now is the evolution of this spin structure with increasing number of bilayers. In order to investigate the long-range order between the moments in the Fe layers, a multilayer stack consisting of 15 bilayers $([\mathrm{Fe}(1.7 \mathrm{~nm}) / \mathrm{Fe}$ oxide $(1.6 \mathrm{~nm})])$ was prepared with the ${ }^{57} \mathrm{Fe}$ layer embedded in the center of every Fe layer. The electronic reflectivity in Fig. 2(a) shows a pronounced first-order Bragg peak, which arises due to the chemical periodicity. From these data, one can determine the thicknesses of the $\mathrm{Fe}$ and the $\mathrm{Fe}$ oxide that constitute the bilayer with an accuracy of about $0.1 \mathrm{~nm}$. Almost equal thicknesses of the Fe and the Fe-oxide layers lead to a suppression of the second-order peak. For measurement of the nuclear reflectivity (i.e., the time-integrated delayed photons within a time window ranging from 12 to $170 \mathrm{~ns}$ after excitation), the sample was aligned with the hard axis of magnetization parallel to the external field and perpendicular to the photon beam direction. The magnetic hard and easy axes were determined via magneto-optical Kerr effect (MOKE) measurements, as shown in Fig. 3(a). The MOKE hysteresis loop for the easy axis is very square with a coercivity of $35 \mathrm{Oe}$, indicating the soft magnetic properties of this system. This anisotropy was most likely induced by the arrangement of the sputter sources within the chamber, leading to an oblique deposition ${ }^{8}$ of the ${ }^{57} \mathrm{Fe}$, while the atoms from the ${ }^{56} \mathrm{Fe}$ target reached the substrate in normal incidence. Nuclear reflectivity curves of the multilayer are shown in Figs. 2(b) and 2(c). In an external field of $\vec{H}=200$ Oe applied perpendicular to $\vec{k}_{0}$ (transverse geometry), additional half-order Bragg peaks appear [Fig. 2(b)], indicating a magnetic periodicity that is twice as large as the chemical one. In the case of NRS, these

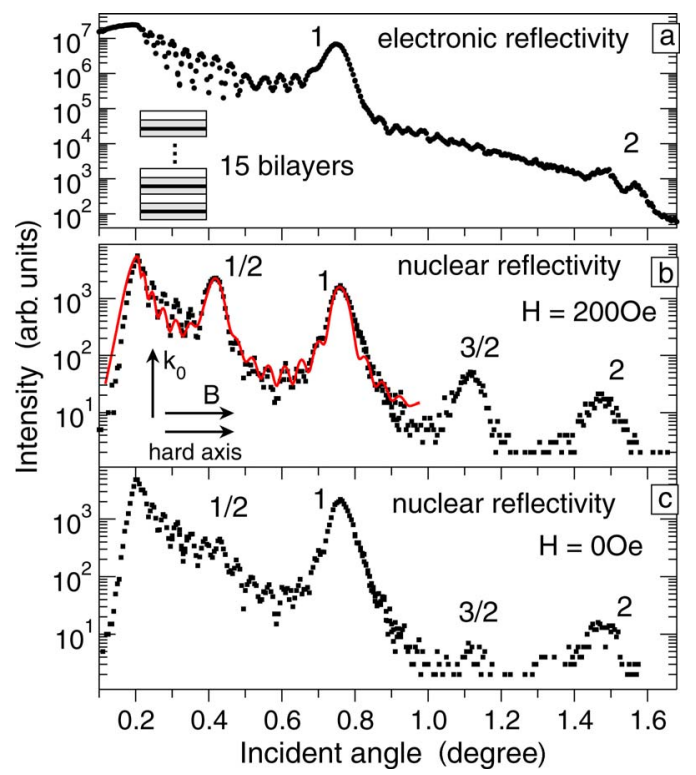

FIG. 2. (Color online) (a) Electronic and [(b) and (c)] nuclear reflectivity curves of the $\mathrm{Fe} / \mathrm{Fe}$-oxide multilayer. In the nuclear reflectivity (b), half-order Bragg peaks appear due to a noncollinear arrangement of the magnetic moments in adjacent iron layers. In the scattering geometry used here (arrows), these peaks vanish for zero field (c), proving their pure magnetic origin. The solid line in (b) is a simulation.

half-order peaks are observed if the projection of the magnetic moments of subsequent $\mathrm{Fe}$ layers on the direction of $\vec{k}_{0}$ is different, as it could be the case for two canted moments. The difference of these projections determines the intensity of the half-order peaks which would reach its maximum value for an antiparallel alignment of the moments along $\vec{k}_{0}$. In remanence, only a small residue of the half-order peak remains [Fig. 2(c)]. Obviously, a reorientation of the mo-

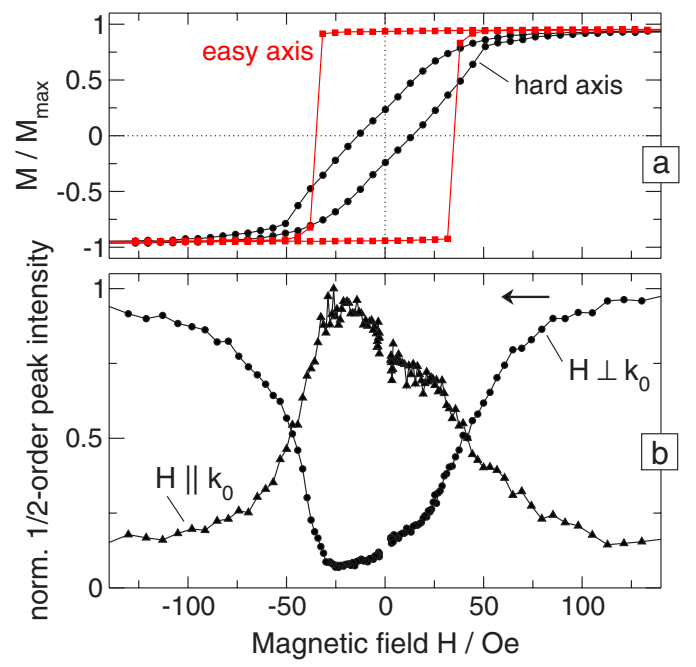

FIG. 3. (Color online) (a) MOKE hysteresis loops for easy and hard axes of the $\mathrm{Fe} / \mathrm{Fe}$ oxide multilayer. (b) Field dependence of the $1 / 2$-order peak for the magnetic field perpendicular and parallel to the incident photon beam. The hard axis was, in both cases, parallel to the external field. 
ments has taken place where the difference in the projections of the moments on the direction of $\vec{k}_{0}$ vanishes. The halforder peaks also disappear if the sample, together with the external field, is rotated azimuthally by $90^{\circ}$, so that $\vec{H}$ is parallel to $\vec{k}_{0}$ (longitudinal geometry). These observations prove the purely magnetic origin of the half-order peaks. Moreover, one can conclude that the canting of the successive iron moments must be symmetric around the direction of the external magnetic field. From the simulation of the nuclear reflectivity shown in Fig. 2(b), we derive a canting angle of about $85^{\circ}$ between adjacent iron layers.

Monitoring the intensity of the magnetic $1 / 2$-order peak, we investigate the low-field properties and the switching behavior of the coupled iron layer spins. This has been done in longitudinal $(\vec{H} \| \vec{k})$ and transverse $(\vec{H} \perp \vec{k})$ geometries with the hard axis parallel to the external field. The respective curves are presented in Fig. 3(b). In the transverse geometry, the 1/2-order peak intensity drops with decreasing field and reaches again its maximum value after field reversal. This behavior can be attributed to a rotation of the coupled moments as one unit with a fixed canting angle. The minimum position at -30 Oe then corresponds to the coercive field where the magnetic moments are symmetric about $\vec{k}_{0}$. However, the measured field dependence of the 1/2-order peak intensity could also be explained if the magnetization of the iron layers breaks up into randomly oriented domains in low fields. To rule out this interpretation, we measured the field dependence also in longitudinal geometry. In the case of domain formation, one does not expect any change in the 1/2-order signal with external field. The measured curve, however, clearly shows a pronounced maximum for small field values, confirming the coherent rotation of the canted moments as a single domain. The absence of a domain structure is also supported by the analysis of the diffuse NRS. Its angular distribution does not show any sign of lateral magnetic inhomogeneities that are smaller than the resolution limit of about $60 \mu \mathrm{m}$.

With further increasing fields, the $1 / 2$-order peak intensity decreases gradually, as shown in Fig. 4, and reversibly recovers upon releasing the external field back to zero. The inset in Fig. 4 shows the longitudinal magnetization of the sample as determined via MOKE magnetometry. The shape of the curve is in accordance with the model presented here: While the steep rise at low fields is due to the rotation of the effective moment toward the external field, the slow increase toward high fields reflects the gradual alignment of the two coupled moments, very similar to the case of a coupled trilayer as demonstrated in Ref. 9. The pictograms in Fig. 4 summarize the orientation and the relative coupling angle of the two sets of moments in the sample. The field dependence of the coupling angle as derived from the 1/2-order peak intensity is shown in Fig. 6 and will be discussed later.

\section{DISCUSSION}

It is clear that the mechanism leading to the observed coupling strongly depends on the properties of the native Fe-oxide layer. In the literature, one finds a lively debate on

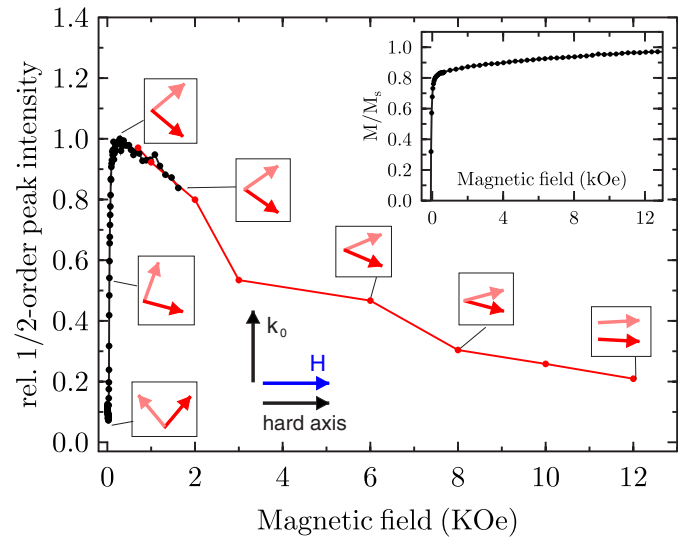

FIG. 4. (Color online) Intensity of the 1/2-order peak as a function of the external magnetic field in a geometry given by the arrows. Pictograms indicate the orientation of the system of coupled moments. The inset shows the positive branch of a longitudinal MOKE magnetization curve of this sample.

the nature of such oxide layers, ${ }^{10-15}$ the structure of which is governed by the crystalline orientation of the surface, the temperature, and the oxygen pressure. In our case, the situation is even more complex due to the polycrystalline structure of the Fe metal from which the oxide originates. Recent studies have shown that buried native oxide layers are intrinsically different from any known surface or bulk Fe oxides. ${ }^{2}$ These layers appear to be ferromagnetically ordered, consisting of two different components, one carrying no magnetic moment like an antiferromagnet and the other exhibiting ferrimagnetic order with an anomalously high moment. In order to reveal the chemical nature of the oxide, we performed x-ray absorption measurements at the DORIS storage ring (DESY, Hamburg). The normalized absorption coefficient of a $5 \mathrm{~nm}$ thick Fe-oxide layer on $\mathrm{Si}$ (produced by multiple deposition of $\mathrm{Fe}$ and subsequent oxidation) is shown in Fig. 5. The dashed lines are ab initio self-consistent multiple scattering calculations of $\mathrm{x}$-ray absorption for $\mathrm{FeO}$ and $\mathrm{Fe}_{3} \mathrm{O}_{4}$ by

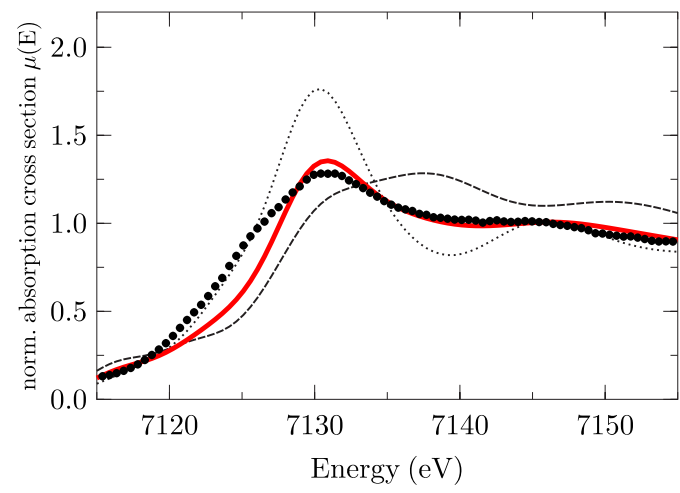

FIG. 5. (Color online) Normalized x-ray absorption coefficient of a $5 \mathrm{~nm}$ Fe-oxide layer on $\mathrm{Si}$, produced via multiple deposition of $\mathrm{Fe}$ and subsequent oxidation under the same conditions as applied for production of the $\mathrm{Fe} / \mathrm{Fe}$-oxide multilayer. The dashed and dotted lines are theoretical calculations for $\mathrm{FeO}$ and $\mathrm{Fe}_{3} \mathrm{O}_{4}$, respectively, based on the FEFF code for x-ray absorption (Ref. 16). The solid red line results from a 1:1 superposition of both calculated curves. 
employing the FEFF code. ${ }^{16}$ The basic shape of the measured curve is reproduced by assuming a mixture of these oxides in a ratio of roughly $1: 1$. The contribution of $\mathrm{Fe}_{2} \mathrm{O}_{3}$ to the measured data seems to be negligible. The remaining deviations in the regime around the absorption edge are most probably due to variations in the chemical state of the oxide in combination with structural disorder that render it to be nonstoichiometric. Although the nature of the oxide is not fully resolved at this stage, these data provide enough evidence to conclude that the oxide contains a significant fraction of antiferromagnetically ordered Fe spins.

These observations lead us to the conclusion that the origin of the magnetic interlayer coupling observed here is most likely a mechanism that was suggested by Slonczewski. ${ }^{17}$ In this model, an antiferromagnetic spacer layer mediates the coupling between two ferromagnetic layers via the shortrange Heisenberg exchange coupling. Assuming perfectly flat, uncompensated interfaces of the spacer layer, one would obtain either ferromagnetic or antiferromagnetic coupling depending on the number of lattice planes in the spacer layer. Lateral variations of the thickness, however, lead to a competition between ferro- and antiferromagnetic coupling, that results in a noncollinear coupling between the adjacent ferromagnetic layers, as reported previously for coupled trilayers, ${ }^{9,18,19} \mathrm{Fe} / \mathrm{Cr}$ multilayers, ${ }^{20}$ and $\mathrm{Fe}_{3} \mathrm{O}_{4} / \mathrm{CoO}$ superlattices. ${ }^{21}$ The equilibrium configuration is given by the local minima of the magnetic free energy $F=F_{c}+F_{a}$ $-\vec{H} \cdot\left(\vec{M}_{1}+\vec{M}_{2}\right) d_{\mathrm{Fe}}$, where $F_{c}$ is the coupling energy density, $F_{a}$ is the anisotropy energy density, and the last term describes the Zeeman energy of the coupled moments $\vec{M}_{1}$ and $\vec{M}_{2}$ in the external field $\vec{H}$ with $d_{\mathrm{Fe}}$ being the thickness of the $\mathrm{Fe}$ layers. According to Slonczewski, the coupling energy has the form

$$
F_{c}=C_{+} \theta^{2}+C_{-}(\theta-\pi)^{2},
$$

where $\theta$ is the angle between the magnetizations of adjacent layers. If the constants $C_{+}$and $C_{-}$are of equal magnitude, orthogonal coupling of the moments is favored. This description is in contrast to models that rely on bilinear and/or biquadratic coupling based on RKKY-type interaction between adjacent layers, where the coupling energy is given by

$$
F_{c}=-J_{B L} \cos \theta-J_{B Q} \cos ^{2} \theta,
$$

with $J_{B L}$ and $J_{B Q}$ being the constants for bilinear and biquadratic couplings, respectively. Therefore, a fit of the experimental data with these two models should allow us to distinguish between them. In fact, a fit with Eq. (1) (solid line in Fig. 6) leads to a very good agreement with the data. For $C_{+}$ and $C_{-}$, we obtain the values of 0.122 and $0.086 \mathrm{erg} / \mathrm{cm}^{2}$, respectively. These values are in the same range as those found for some previously studied trilayer systems. ${ }^{9,18}$ On the other hand, the best fit with Eq. (2), shown as a dashed line, significantly deviates from the measured data. This strongly supports the validity of the Slonczewski model in our case.

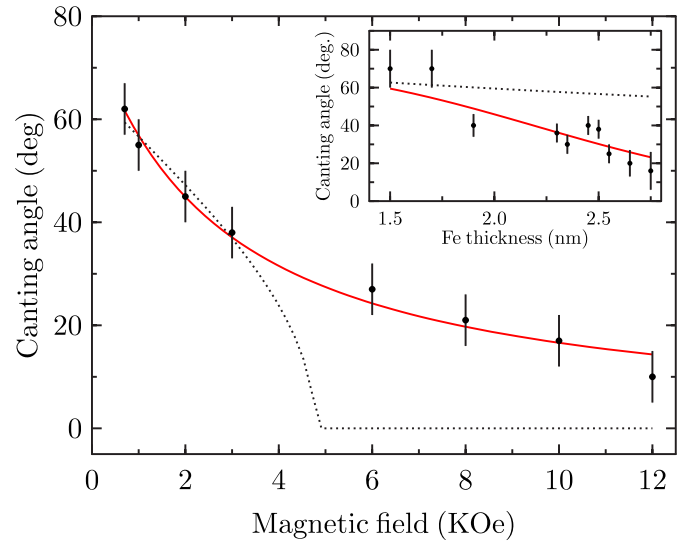

FIG. 6. (Color online) Coupling angle between the two magnetic $\mathrm{Fe}$ sublattices in the $\mathrm{Fe} / \mathrm{Fe}$-oxide multilayer. The solid line is a fit according to the Slonczewski model, and the dashed line is the best fit obtained assuming bilinear and/or biquadratic coupling. The inset shows the thickness dependence of the coupling angle for an external field of $700 \mathrm{Oe}$, where the solid line is a visual guide while the dashed line is a calculation according to Eq. (1).

More details about the coupling mechanism could be revealed via the thickness dependence of the magnetic structure. While the Fe-oxide thickness is difficult to control in case of such polycrystalline systems, we have investigated the magnetic coupling here as a function of Fe layer thickness $d_{\mathrm{Fe}}$. The inset of Fig. 6 displays the low-field canting angle as it decreases with increasing $d_{\mathrm{Fe}}$. This dependence cannot be described by the Zeeman term in the magnetic free energy alone, because it leads only to a weak thickness dependence (dashed line). It is clear that the model introduced here has to be refined to take also into account the ferrimagnetic part of the oxide (contributed by the $\mathrm{Fe}_{3} \mathrm{O}_{4}$ ) that participates in the coupling. For that reason, the microscopic structure of the native oxide layers still has to be explored.

In conclusion, using NRS, we were able to directly observe a noncollinear coupling of polycrystalline iron layers through a native oxide spacer. This coupling seems to be mediated by antiferromagnetically ordered components in the oxide. The canting angle between adjacent layers is close to $90^{\circ}$ as observed before for some single-crystalline systems. Remarkably, the coupling persists over a large number of $\mathrm{Fe} / \mathrm{Fe}$-oxide bilayers, giving rise to a long-range magnetic correlation in a multilayer that is stable up to rather high fields beyond $12 \mathrm{kOe}$. This easy-to-prepare system may open interesting perspectives for applications in magnetoengineering and related fields.

\section{ACKNOWLEDGMENTS}

Sincere thanks go to R. Rüffer, S. Stankov, K. Schlage, and $\mathrm{T}$. Klein for their assistance during the experiments at ESRF, to K. Saksl, E. Welter, and O. Seeck for their support at HASYLAB, and to J. Korecki for helpful discussions. We gratefully acknowledge K. Saksl for providing the FEFF calculations for the Fe oxides. This work was supported by the European Commission under FP6 Contract No. NMP4-CT2003-001516 (DYNASYNC). 
${ }^{1}$ R. E. Camley and R. L. Stamps, J. Phys.: Condens. Matter 5, 3727 (1993).

${ }^{2}$ G. S. D. Beach, F. T. Parker, D. J. Smith, P. A. Crozier, and A. E. Berkowitz, Phys. Rev. Lett. 91, 267201 (2003).

${ }^{3}$ L. Niesen, A. Mugarza, M. F. Rosu, R. Coehoorn, R. M. Jungblut, F. Roozeboom, A. Q. R. Baron, A. I. Chumakov, and R. Rüffer, Phys. Rev. B 58, 8590 (1998).

${ }^{4}$ C. L'abbé, J. Meersschaut, W. Sturhahn, J. S. Jiang, T. S. Toellner, E. E. Alp, and S. D. Bader, Phys. Rev. Lett. 93, 037201 (2004).

${ }^{5}$ R. Röhlsberger, H. Thomas, K. Schlage, E. Burkel, O. Leupold, and R. Rüffer, Phys. Rev. Lett. 89, 237201 (2002).

${ }^{6}$ T. S. Toellner, W. Sturhahn, R. Röhlsberger, E. E. Alp, C. H. Sowers, and E. E. Fullerton, Phys. Rev. Lett. 74, 3475 (1995).

${ }^{7}$ R. Rüffer and A. I. Chumakov, Hyperfine Interact. 97/98, 589 (1996).

${ }^{8}$ M. T. Umlor, Appl. Phys. Lett. 87, 082505 (2005).

${ }^{9}$ P. A. A. van der Heijden, C. H. W. Swüste, W. J. M. de Jonge, J. M. Gaines, J. T. W. M. van Eemeren, and K. M. Schep, Phys. Rev. Lett. 82, 1020 (1999).

${ }^{10}$ G. W. R. Leibbrandt, G. Hoogers, and F. H. P. M. Habraken,
Phys. Rev. Lett. 68, 1947 (1992).

${ }^{11}$ S. J. Roosendaal, A. M. Vredenberg, and F. H. P. M. Habraken, Phys. Rev. Lett. 84, 3366 (2000).

${ }^{12}$ H.-J. Kim, J.-H. Park, and E. Vescovo, Phys. Rev. B 61, 15288 (2000).

${ }^{13}$ K. Mori, M. Yamazaki, T. Hiraki, H. Matsuyama, and K. Koike, Phys. Rev. B 72, 014418 (2005).

${ }^{14}$ K. Mori, M. Yamazaki, T. Hiraki, H. Matsuyama, and K. Koike, Phys. Rev. B 74, 026405 (2006).

${ }^{15}$ E. Vescovo, Phys. Rev. B 74, 026406 (2006).

${ }^{16}$ A. L. Ankudinov, B. Ravel, J. J. Rehr, and S. D. Conradson, Phys. Rev. B 58, 7565 (1998).

${ }^{17}$ J. C. Slonczewski, J. Magn. Magn. Mater. 150, 13 (1995).

${ }^{18}$ M. E. Filipkowski, J. J. Krebs, G. A. Prinz, and C. J. Gutierrez, Phys. Rev. Lett. 75, 1847 (1995).

${ }^{19}$ C.-H. Lai and K. H. Lu, J. Appl. Phys. 93, 8412 (2003).

${ }^{20}$ A. Schreyer, J. F. Ankner, Th. Zeidler, H. Zabel, C. F. Majkrzak, M. Schäfer, and P. Grünberg, Europhys. Lett. 32, 595 (1995).

${ }^{21}$ Y. Ijiri, J. A. Borchers, R. W. Erwin, S.-H. Lee, P. J. van der Zaag, and R. M. Wolf, J. Appl. Phys. 83, 6882 (1998). 